\title{
Physical therapy for temporomandibular disorders: evidence-based improvements and enhancements for diagnosis and management
}

\author{
Janey Prodoehl ${ }^{1}$, Emily Kahnert ${ }^{2}$ \\ ${ }^{1}$ Physical Therapy Program, Midwestern University, Downers Grove, IL, USA; ${ }^{2}$ TMD, Orofacial Pain \& Dental Sleep Medicine Clinic, School of \\ Dentistry, University of Minnesota, Minneapolis, MN, USA \\ Contributions: (I) Conception and design: Both authors; (II) Administrative support: Both authors; (III) Provision of study materials or patients: None; \\ (IV) Collection and assembly of data: None; (V) Data analysis and interpretation: None; (VI) Manuscript writing: Both authors; (VII) Final approval \\ of manuscript: Both authors. \\ Correspondence to: Janey Prodoehl, PT, PhD, CCTT. Physical Therapy Program, Midwestern University, 555 31st Street, Downers Grove, IL 60515, \\ USA. Email: jprodo@midwestern.edu.
}

\begin{abstract}
The most common oral and maxillofacial disorders treated by physical therapists are temporomandibular disorders (TMD), and rehabilitation strategies continue to evolve with advancements in research and technology. While physical therapy is an evidence-supported approach to the management of TMD, difficulties with referral to and access to appropriately trained physical therapists can create disparity in care for patients. Lack of interdisciplinary collaboration and practice is an ongoing issue, particularly among dentists and physical therapists and this can translate to less than optimal care for individuals with TMD. While dentists and physical therapists may utilize similar diagnostic criteria, implementation gaps in use of consistent diagnostic criteria still exist across both professions. Additionally, possibilities exist for the future expansion of such criteria to recognize the contribution of structures and comorbidities outside of the masticatory system such as the cervical spine and central nervous system changes that can promote the persistence of pain in some individuals with TMD. Pain neuroscience education, epigenetics, and other rehabilitation tools such as virtual reality may allow physical therapists to address the central nervous system changes associated with pain persistence and a fear of movement seen in some individuals with TMD. Applying biomechanical knowledge gained from real-time ultrasound muscle imaging and musculoskeletal modeling will enhance diagnostic management and post-surgical rehabilitation approaches. The purpose of this review paper is to describe novel evidence-based rehabilitative tools or concepts that can be used to improve the diagnosis and management of individuals with TMD. Continued growth and development in research and clinical practice related to TMD will ultimately lead to improved care for individuals with TMD, both conservatively and post-surgically.
\end{abstract}

Keywords: Rehabilitation; physical therapists; dentists; orofacial pain; facial pain; interdisciplinary care; physical medicine

Received: 22 July 2020; Accepted: 31 May 2021; Published: 10 June 2022.

doi: $10.21037 /$ fomm-20-44

View this article at: http://dx.doi.org/10.21037/fomm-20-44 


\section{Introduction}

The most common oral and maxillofacial disorders treated by physical therapists are temporomandibular disorders (TMD), and rehabilitation strategies continue to evolve with advancements in research and technology. The diverse conditions included under the TMD heading are complex and can greatly impact an individual's quality of life. In general, physical therapy aims to prevent, correct and/or alleviate movement dysfunction which commonly interferes with eating, talking, yawning and chewing in individuals with TMD. Physical therapy is well recognized as a conservative method for the management of symptoms associated with TMD (1-4). However, difficulties with referral to and availability of appropriately trained physical therapists can create disparity in care (5). Lack of interdisciplinary collaboration and practice is an ongoing issue, particularly among dentists and physical therapists. This can translate to less than optimal care of individuals with TMD. By promoting innovation in care and interdisciplinary collaboration, the management of individuals with TMD will continue to progress with exciting potential for improvement in conservative and preand post-operative rehabilitation.

The need for improved interdisciplinary collaboration and prioritized TMD research were two of many care gaps recently highlighted in the 2020 report of the National Academies of Science, Engineering and Medicine on Temporomandibular Disorders: Priorities for Research and Care (5). The report identified poorly coordinated care as a contributing factor to TMD overtreatment, and called for improved evidence for physical therapy interventions in discussing physical therapy as one component of conservative management (5). Expanding existing clinical and biomechanical research to the temporomandibular joint (TMJ) will contribute additional knowledge of joint behavior and stability over time to inform rehabilitation and surgical protocols (6-8). Increasing recognition of the role of structured physical therapy in improving postsurgical patient outcomes lends support to interprofessional collaboration for improving patient outcomes following TMJ surgery $(9,10)$. However, education of healthcare professionals within each discipline involved in the care of individuals with TMD must expand beyond its current state of training to meet the inter- and multi-disciplinary needs of this patient population $(5,11,12)$.

The current international diagnostic standard for clinical and research application in individuals with TMD is the dual-axis Diagnostic Criteria for TMD (DC/TMD) $(13,14)$. The DC/TMD includes validated physical (Axis I) diagnostic algorithms and consideration of psychosocial status and pain-related disability (Axis II). The DC/TMD progressed diagnostic capabilities of clinicians and provides a consistent classification framework to guide clinical decision-making and research across disciplines. Physical therapists utilize the DC/TMD for classification of TMD symptoms $(15,16)$. However, implementation gaps still exist, with inconsistencies across disciplines, and areas needing refinement. For example, the prevalence of myalgic TMD is greater than that of arthralgic and degenerative causes, yet myogenous involvement in TMD lacks quantitative validation $(14,17,18)$. New avenues supporting identification of myogenous involvement in TMD would therefore be helpful.

Another opportunity for refinement concerns the contribution of structures and systems outside of the masticatory system such as the cervical spine and central nervous system which can contribute to the persistence of pain in individuals with TMD. The connection between the cervical spine and TMD is well-documented in the literature (19-25) but is minimally addressed in the dentistry-focused DC/TMD. Similarly, the central nervous system provides an accessible link between DC/TMD Axis I and II symptoms without guidance for inclusion in rehabilitation. As the understanding of the biological mechanisms of persistent pain in other musculoskeletal conditions such as chronic low back pain has grown, novel approaches to improving movement dysfunction and pain in individuals with chronic TMD are emerging. Therapeutic pain neuroscience education (PNE), epigenetics, and other rehabilitation tools such as virtual reality allow physical therapists to address the central nervous system changes associated with pain persistence and fear of movement in individuals with chronic pain. Expanding diagnosis and management to include these areas will greatly improve the ability of physical therapists to implement effective rehabilitation strategies for individuals with TMD.

The purpose of this review is to describe novel evidencebased rehabilitative tools or concepts that can be used to improve the diagnosis and management of individuals with TMD. Specifically, we will (I) review future directions in interdisciplinary care and collaboration, (II) discuss expansion of existing diagnostic considerations to account for the cervical spine and improved muscle imaging with real time ultrasound, (III) explore applications of musculoskeletal modeling in the study of in-vivo TMJ 
biomechanics, (IV) describe implications for TMJ preand post-surgical physical therapy, and (V) discuss the incorporation of therapeutic PNE and epigenetics to address central sensitization in individuals with persistent TMD pain.

\section{Interdisciplinary practice}

\section{The interdisciplinary team in the diagnosis and management of TMD}

Increased recognition of the biopsychosocial factors associated with TMD has led to support for an interdisciplinary biopsychosocial model of TMD that is focused on an individual's health and well-being beyond structures in the head and face (5). Some patients with chronic TMD may have significant underlying comorbidities which require specific intervention from a trained psychologist. For example, a relationship has been shown between post-traumatic stress disorder and chronic pain incidence (26). Failure to identify these comorbidities and seek appropriate resources for these individuals is a concern. However, for the majority of individuals with TMD, an interdisciplinary approach to the management of individuals with TMD is recommended $(4,11,12)$.

One of the challenges to achieving an interdisciplinary approach in clinical practice is the varied availability of appropriately trained professionals who can work effectively in an interdisciplinary manner. An interdisciplinary team for individuals with chronic TMD may consist of dentists, physical therapists, psychologists, and physicians, but interdisciplinary care coordination does not always occur. Clearly understanding the role of each healthcare professional and working effectively together with good communication promotes safe, quality health care (27). Too often in healthcare, including the care of individuals with TMD, each discipline operates in a silo, and neither clinical nor research efforts are sufficiently collaborative. Yet in individuals with orofacial pain, outcomes have been shown to improve with an interdisciplinary program approach including a dentist, psychologist, and physical therapist (28).

\section{Interprofessional collaboration and training}

One of the barriers to excellence in interprofessional practice is lack of recognition of the role of other professions and the need for interprofessional practice by each discipline involved in the care of individuals with TMD. An example of this are the results of a recent survey of dentists in Florida which indicated that $41 \%$ of respondent dentists were not aware that physical therapists can treat TMD (19) despite the recognition of physical therapy as a recommended evidence based conservative treatment for TMD. Lack of recognition of physical therapy as an appropriate option for individual with TMD by some healthcare professionals must be addressed to improve access of care to individuals with TMD. Differences in international standards for each profession can also be a barrier to effective collaboration. A recent study of Filipino dentists and physical therapists showed low education and knowledge among dentists and physical therapists (20). Looking to the future, more practical opportunities for interprofessional training and collaboration among physical therapists, dentists and physicians during both pre-doctoral education and post-doctoral practice is one way to address such discrepancies.

One factor that may contribute to lack of understanding of the role of different health professions in the management of individuals with TMD are differences in the scope of practice and training of healthcare practitioners regionally across the US and in different parts of the world. Dental schools in the US with an orofacial pain program may provide more training to student dentists on interdisciplinary care compared to those programs without these resources (21). Similarly, not all physical therapy education programs have equal levels of entry level training related to TMD content (22), and nor are all physical therapists confident in treating this patient population (23). In the US, all 50 states, the District of Columbia, and the US Virgin Islands allow patients to seek some level of treatment from a licensed physical therapist without a referral (24). This level of access has been made possible by elevating entry-level educational standards of physical therapists in the US to the doctoral level, including additional differential diagnosis and medical screening training to ensure patient safety. Moving forward, physical therapists must do a better job communicating and collaborating with dentists and physicians to establish their role in the interdisciplinary team. Challenges exist at the international level however where discrepancies in training standards across disciplines lead to difficulty applying evidence into practice. Looking to the future, a clear standard of care is needed to ensure that rehabilitation efforts for care of individuals with TMD are comprehensive, evidence based and collaborative. To promote team based collaborative practice, interprofessional attitudes 
among healthcare disciplines which can negatively impact communication and collaboration must be modified and addressed (27).

\section{Expanding diagnostic considerations}

\section{Cervical spine and TMD}

Physical therapists include examination of the cervical spine in their examination of individuals with TMD. Individuals with TMD have been shown to have signs of upper cervical spine movement impairment which can be greater in those with headache complaints (25). Silveira et al. (29) found a strong correlation between jaw dysfunction and neck disability. In addition, tender cervical points in individuals with TMD have been found in multiple studies (29-32). A recent Delphi study of international expert TMD physical therapists recognized that manually screening the cervical spine and testing neck muscle function in addition to examining masticatory muscles and jaw movements may improve clinical evaluation of pain and dysfunction in individuals with TMD (33). A randomized controlled trial in women with TMD found that treatment aimed at improving upper cervical spine mobility improved orofacial pain and headache after 5 weeks of treatment (34). Moving forward, appropriately screening for contributing or concomitant cervical involvement should be included in examination of individuals with TMD in order to fully understand the impairments that may contribute to or perpetuate TMD symptoms. This is an area where dentists and physical therapists must work together in order to provide appropriate treatment for their patients. As physical therapists must be trained to recognize the need for an oral appliance and appropriately refer a patient to a dentist, a dentist should be trained to recognize a cervical component to TMD symptoms and appropriately refer a patient to a physical therapist.

\section{Real-time ultrasound (RTUS) used for TMF and muscle imaging}

Long understood as a modality for imaging in other regions, real-time musculoskeletal ultrasound imaging has recently emerged as a practical, accessible modality for clinical assessment in rehabilitation. While magnetic resonance imaging (MRI) is the gold standard technique for diagnosing TMJ disc displacement and degeneration (14), MRI has been shown to identify nearly one-third of asymptomatic subjects with TMJ disc displacement (35-37) and MRI findings alone cannot identify a condition that needs treatment from that which does not. Considering the cost and inconvenience of MRI, the rate of false positive findings negates its usefulness in routine clinical practice.

RTUS imaging is one imaging modality that holds promise for both the diagnosis of and management guidance of joint and muscle dysfunction for individuals with TMD. RTUS imaging is used by physical therapists to document changes in the clinical condition, identify when to progress exercises or activities, localize specific targets for intervention, guide needle placement for trigger point dry needling or electroneuromyography, and to help guide clinical decision making relative to prognosis and progression based on tissue structure (38). RTUS has shown good intra- and inter-rater reliability for use in assessing joint translation at the shoulder, even for a physical therapist with minimal RTUS training (39). In the TMJ, Ho et al. (40) determined that RTUS can reliably image anterior condylar translation, reporting a linear relationship between translation and mouth opening which could aid clinical estimation of rehabilitation potential. In combination with providing opportunities for improved patient education, RTUS can improve diagnosis and management for individuals with TMD.

Assessing myogenous disorders has inherent challenges as shown by the lack of sensitivity and specificity data for these diagnoses (14). The overlapping symptoms often seen in individuals with joint and muscle TMD require accurate physical therapy diagnosis to maximize rehabilitation outcomes. Real time ultrasound can assess skeletal muscle, and in combination with visualization of condylar translation data, can identify muscle disorders more accurately. RTUS can reveal muscle characteristics such as cross-sectional area, thickness, fascicle length and pennation angle (41), and has been used to quantify muscle function in neuromuscular disorders (42). Combining muscle cross-sectional area with condylar translation data could quantify muscle function in joint disorders, which may improve diagnostic accuracy and help predict postsurgical rehabilitation responses. Overstretching muscles already in spasm can negatively impact recovery, so reliably improving clinical assessment accuracy is of great interest for these patients and their providers. The ability to assess muscle behavior with RTUS will improve treatment planning and adaptation for this group of disorders that has been previously difficult to quantify.

An emerging ultrasound-based modality with additional 
clinical applications is shear wave elastography (SWE) which yields information regarding musculoskeletal tissue properties. By measuring the speed of shear-wave propagation through muscle and tendon, SWE can quantify tissue stiffness and integrity $(43,44)$ which has implications for myogenous forms of TMD. This modality has shown good repeatability and accuracy in shoulder muscle and tendon (43) and has been used to examine changes in muscle stiffness in individuals with and without chronic neck pain $(45,46)$. RTUS and SWE has good potential with future refinements to facilitate diagnosis of myofascial syndromes and tendonitis, and their use will allow therapists to monitor tissue healing after surgical intervention throughout the course of rehabilitation.

One specific clinical use of RTUS and SWE is identification of myofascial trigger points (MTrPs). In a 2018 narrative review, Do and colleagues reported ultrasound as having potential to diagnose MTrPs in subjects with migraine and tension-type headache (47). Turo et al. (44) studied MTrPs in the upper trapezii of patients with chronic neck pain and found that the trigger points were identifiable as focal dark areas on ultrasound images. Using SWE, Turo et al. demonstrated that trigger point areas were stiffer, allowing for physical localization of the trigger point. In orofacial pain conditions, correlating MTrPs with subjective symptom report will help quantify and differentiate between myofascial conditions such as myalgia and muscle spasm. Localizing trigger points will improve treatment efficacy, allowing for direct application of manual release work or dry needling. Overall, real time ultrasound is an accessible, reliable, non-invasive diagnostic tool with potential for future widespread clinical use in the assessment and management of TMD.

\section{In-vivo modeling of TMJ biomechanics}

In the TMJ, knowledge regarding the development and progression of disc disorders has continued to evolve. Schiffman et al. (6) recently assessed the longitudinal stability of disc disorders and degenerative joint disease with advanced imaging after 8 years and found that the majority of subjects showed no change over time, with the remainder divided between progression and reversal of their conditions. These types of studies are important for improved understanding of long-term joint behavior yet difficult to execute due to the time and resources required for completion. Musculoskeletal modeling consists of building a $3 \mathrm{D}$ biomechanical model with tissue material properties and muscle force and activation information as inputs (48). Modeling allows for assessment of effects due to variations that would be difficult to measure in reallife such as changing anatomical conditions and testing small differences in movement. In a recent finite element modeling study, Dixit et al. used musculoskeletal modeling to demonstrate long term glenohumeral joint and muscular changes in the presence of simulated injury conditions (49). In combination with other in-vivo measures, a similar approach to modeling the TMJ could predict disc and joint behavior over time for use in determining TMD rehabilitation strategies.

Correctly classifying the kinetics and kinematics of movement is essential to provide good clinical care, and modeling has potential to advance knowledge in this area. In the shoulder, etiology of subacromial impingement has been studied via 3D bone modeling created from subjectspecific fluoroscopic and MRI data (50,51). Modeling revealed that traditional beliefs regarding shoulder impingement throughout shoulder elevation were not replicated anatomically, and that pain is likely due to other mechanisms or structures (50,51). A 2015 knee modeling study used a subject-specific musculoskeletal model informed by data from an implant placed during an instrumented total knee arthroplasty (52). The study demonstrated that modeling produced accurate estimations of knee kinematics which could aid future implant design and inform individualized surgical protocols (52). In the TMJ and articular disc, such modeling would allow for detailed study of movement and injury processes to inform diagnostic and treatment protocols. Varying muscle activation inputs, disc position, and anatomical details such as joint surface characteristics and forces will yield detailed and accurate functional information that can guide surgical and rehabilitation decision-making.

One such musculoskeletal model of the TMJ has been created by Tuijt and colleagues for the purpose of assessing anatomical conditions that cause open locking (7). The model included twenty-four jaw muscles with muscle moments and force capacity estimates as inputs to the model (8). By varying the angle of the articular eminence in the model, Tuijt and colleagues were able to assess the efficacy of different muscular strategies to reduce open locking in different anatomical conditions (8). These modeling studies provide clinical support for rehabilitation interventions such as training muscle control during movement and teaching lock reduction strategies. They also suggest that presurgical subject-specific modeling could also help determine 
the ideal articular eminectomy approach for a patient with respect to their existing anatomy and muscle control $(7,8)$. In the future, this same approach can be applied to other disc displacement and masticatory muscle disorders, and the results of modeling studies may be useful when making informed decisions regarding TMJ surgery and rehabilitation protocols.

\section{Pre- and post-surgical rehabilitation and management}

When considering TMJ surgery, a conservative approach consisting of pain management and rehabilitation is the first line recommended treatment for individuals with TMD $(53,54)$. For individuals who fail a conservative approach or who are appropriate for direct surgical intervention, post-surgical management is typically more defined in its interprofessional approach with a team led by the oral surgeon and including physical therapists, physicians and pharmacists. However, effective collaborative practice for post-surgical patients often relies on the oral surgeon to have developed interprofessional collaborations and communication with other team members. Given the varied level of entry level training among physical therapists alone on post-surgical management of individuals with TMD (22), it can be challenging for the oral surgeon to find an appropriately trained physical therapist for collaboration.

There is strong evidential support for the use of physical therapy after open TMJ surgery to achieve good postoperative outcomes (9). Additionally, there is evidence to support better functional patient outcomes in patients' status post condylar discopexy with early supervised physical therapy compared to self-directed home exercise (10). Consultation with a specialized pain clinic has also been shown to reduce pain after oral and maxillofacial surgery (55). Additionally, preoperative physical therapy, or prehabilitation, has been shown to lead to improved physical and functional recovery immediately postoperatively in some orthopedic surgeries including total knee arthroplasty (56) and hip fracture (57). However, preoperative physical therapy may not affect longer term outcomes following joint replacement surgery (58). Investigation of the effects of preoperative physical therapy in modifying outcomes following TMD surgery has not yet been undertaken. Looking to the future, a healthcare system that embraces and rewards interprofessional practice both conservatively and post surgically to promote excellence in patient outcomes would be a goal to best serve all patients.

\section{Addressing central sensitization in TMD}

Pain has a multidimensional nature that includes physiological, sensory, affective, cognitive, behavioral, and social/cultural/spiritual/political aspects (59). Activation of pain pathways in the central nervous system is expected when a noxious input is applied at the periphery (60). In the case of TMD, peripheral input is expected to come from masticatory muscles or TMJ structures. However, this pain model does not explain the persistence or magnitude of pain in individuals with chronic TMD. Individuals with different forms of TMD may be more likely to have persistent pain. For example, $31 \%$ of individuals with a myalgic form of TMD at baseline continued to have their disorder over a 5 -year period (61).

There is evidence to support sensitization of the central nervous system as an underlying mechanism explaining the persistence of pain in some individuals with chronic TMD (62). The International Association for the Study of Pain recommends distinguishing between different types of pain (i.e., nociceptive, nociplastic, and neuropathic), since the classification impacts assessment and management (59) and TMD can be associated with neuropathic pain syndromes distinguished by both central and peripheral changes (63). Current models of TMD diagnostic classification such as the internationally recognized DC/TMD (14) do not include a clinical assessment of central sensitization as part of the physical examination necessary for the diagnosis of TMD beyond pain referral with applied pressure. However, the presence of allodynia (pain triggered by innocuous stimuli), hyperalgesia, or secondary hyperalgesia (spreading of pain beyond the face) (64) has important implications for management of individuals with TMD. Refinements of diagnostic classification schemes in the future may look to include identification of individuals likely to develop persistent pain to provide a complete clinical picture for management of individuals with chronic TMD. Expecting such patients to achieve good outcomes by considering only the joint and muscle influences using oral appliance or standard physical therapy exercises alone is unrealistic. Additional examination methods may include assessment of pressure pain threshold, thermal sensation, mechanical detection threshold, vibration detection threshold, or twopoint discrimination (63). Use of these assessments for documenting progress and outcomes over time should also be considered in the management of these patients and in guiding decision making regarding surgical intervention. 


\section{Addressing kinesiophobia}

In addition to clarifying the clinical picture, identification of biopsychosocial factors underlying chronic pain can suggest new avenues of treatment. Lira et al. (65) found that patients with TMD and higher levels of kinesiophobia (i.e., fear of movement) showed a more complex clinical TMD presentation with high psychosocial distress, widespread mechanical pain sensitivity, and a more complex presentation of myalgia, disc displacement, and arthralgia (65). Specifically, they found a 13 times greater chance of a complex presentation in individuals with high-kinesiophobia compared to those with moderate-kinesiophobia (65). Fear of movement in the orofacial region can manifest as alterations in functions such as eating, talking or yawning, and there is evidence to support kinesiophobia in chronic TMD (66-69).

Fear of movement and pain-related fear are recognized phenomena in individuals with other forms of persistent musculoskeletal pain including low back pain, where chronicity of pain may be predicted by movementrelated fear (70-73) and neck pain (74,75). An improved understanding of factors which can influence the development of persistent pain has led to new approaches in management of chronic musculoskeletal pain such as virtual reality and externally focused rehabilitation approaches to improve function and reduce movement related fear (76-80). For example, virtual reality activities such as dodgeball or virtual walking have been used to engage individuals with chronic low back to improve spinal mobility (77) and to reduce pain and kinesiophobia (81). Such novel approaches have yet to be investigated in individuals with chronic orofacial pain.

\section{Therapeutic PNE}

In Melzack's neuromatrix theory explaining chronic pain, pain is multidimensional and not simply a response to a noxious input to prevent further injury but a disease itself, "-the result of neural mechanisms gone awry" (71). While activation of the neuromatrix may be triggered by a sensory input as in the case of acute TMD or post-surgical pain, impulses may also be generated independent of any sensory input. In a chronic pain situation, it has been proposed that the pain neuromatrix becomes progressively strengthened via both nociceptive and non-nociceptive inputs (82). Based on the pain neuromatrix concept, Moseley proposed a rehabilitative approach to treating persistent pain (82). This approach includes decreasing threatening input to the system where input may be both nociceptive (e.g., inflammation) and non-nociceptive (e.g., fear of movement) (72). Patient education is a central part of this PNE approach for the management of chronic persistent pain with promise for individuals with persistent TMD.

The goal of PNE is to reduce the perceived threat of pain by increasing a patient's understanding of how the nervous system and body work (82). An example of this is education that the central nervous system has become sensitized, and that while pain itself is normal, the processes behind it can become modified (83). There is strong evidence to support the use of PNE with chronic musculoskeletal pain to reduce pain intensity and disability and to improve pain catastrophization, fear-avoidance, and abnormal pain related behaviors (84). Individuals with chronic pain perceive movement as difficult because the motor cortex is being utilized as part of the pain neuromatrix, thus perpetuating the cycle of pain catastrophization, fear-avoidance, and abnormal pain related behavior unless intervention occurs to break the cycle (85).

Entry level training of physical therapists in the US includes the neuroscience of pain and PNE principles, and PNE delivered by physical therapists has been shown to be effective in the management of individuals with chronic pain (86-88). Adding a single PNE session prior to surgery for lumbar radiculopathy has resulted in significant healthcare savings over 3 years (89). Pre-surgical education for TMD using PNE principles may work to reduce fear and anxiety before surgery and assist in developing realistic expectations regarding pain after surgery as has been shown following spinal surgery (90-93). A recent systematic review supported the use of PNE in the management of chronic musculoskeletal disorders by reducing pain, improving function and lowering disability, reducing psychosocial factors, enhancing movement, and minimizing healthcare utilization (84).

The use of PNE is an avenue that should be explored in individuals with persistent orofacial pain. A recent case report of an individual with a 5 -year history of bilateral chronic myofascial TMD and comorbid neck/right arm pain described the successful use of manual physical therapy together with graded motor imagery, a PNE technique, to reduce kinesiophobia and improve function (94). There is growing evidence related to the use of preoperative PNE programs for improving long term outcomes following some surgeries, but not TMD surgery. While the immediate post-operative outcomes have not been shown to change 
following a single pre-operative education session (95), long term outcomes have been shown to change. A 30-minute preoperative PNE program delivered by a physical therapist significantly impacted health care costs at 1 year in patients who underwent spinal surgery with a reduction of health care expenditure by $45 \%$ compared to a control group (86), and these savings were shown to be maintained at 3 years (89). Use of pre-operative physical therapy for changing patient outcomes and cost of care is an area that needs to be explored for TMJ surgery.

\section{Epigenetics and TMD}

Epigenetic mechanisms are biological processes that change gene expression, and are involved in the complex interaction between genetics and environment behind most chronic conditions (96). While epigenetic processes are required for some normal cell function, there are three site-specific mechanisms of interest for chronic pain: modifications to the histones in DNA structure, DNA methylation, and RNA transcription interference (97). All three of these mechanisms result in genetic modifications that can increase an individual's susceptibility to experiencing the synaptic potentiation associated with plasticity, associative learning and memory, which will change pain thresholds and contribute to neuropathic pain (96). Maladaptive plasticity can create phenomena such as central sensitization, hyperalgesia and allodynia. The connection between TMD and central sensitization is well-documented, and frequently surfaces as a barrier to rehabilitation (62). Therefore, studying epigenetics has potential to contribute new knowledge regarding the biological mechanisms behind chronic pain and management strategies for individuals with chronic orofacial pain.

Epigenetic processes are central to PNE and future research on their application to rehabilitation will guide clinicians as technology evolves. Much of the existing epigenetic research in rehabilitation has focused on rat models, with demonstrated results ranging from exerciseinduced hypoalgesia to decreased neuronal excitability and modulation of neuropathic pain (96). Extending this research for use with PNE in human subjects will allow therapists to maximize the capacity for motor learning within the context of patient-specific rehabilitation needs. By combining epigenetic markers with clinical assessment, prediction of vulnerability to chronic pain after procedures and/or overreaction of the immune system to exercise becomes possible (96). The predominance of chronic pain in orofacial pain disorders makes TMD rehabilitation an especially appropriate area for adapting future epigenetic mechanisms to help manage neuropathic pain and improve the efficacy of exercise programs.

\section{Conclusions}

This review has discussed a range of topics regarding novel evidence-based improvements to physical therapy diagnosis and management of individuals with TMDs. Strengthening interdisciplinary collaboration and education will allow physical therapists to play a vital role in biopsychosocial multidisciplinary care including pre- and post-surgical management. The importance of a correct diagnosis and the prevalence of overlapping conditions in orofacial pain (5) highlights the need for expanded physical therapy diagnostic criteria to address the cervical spine and central nervous system. Similarly, using tools such as RTUS to further identify muscle disorders and applying knowledge of TMJ biomechanics gained from modeling studies could improve treatment efficacy and post-surgical rehabilitation outcomes. Central nervous system changes that can occur in some individuals with TMD can be successfully addressed by physical therapists through novel applications of therapeutic PNE, virtual reality applications, and epigenetic advancements. Without these strategies, barriers such as central sensitization and kinesiophobia threaten to undermine successful pain management and restrict restoration of normal function for some individuals with persistent pain. The strategies discussed in the review are consistent with the recommendations of the 2020 TMD consensus study report which called for additional research, training, and interdisciplinary collaboration to improve clinical care practices for this population (5). While far from exhaustive, the list of subjects covered reveals the vast potential that exists for enhanced rehabilitative care in this area. Innovative, coordinated, evidence-based physical therapy provided alongside colleagues from multiple disciplines will visualize the future of successful rehabilitation for individuals with TMD.

\section{Acknowledgments}

Funding: None.

\section{Footnote}

Provenance and Peer Review: This article was commissioned 
by the Guest Editors (Stephen Feinberg and Louis Mercuri) for the series "Temporomandibular Joint Disorders Diagnosis and Management - What Does the Future Hold?" published in Frontiers of Oral and Maxillofacial Medicine. The article has undergone external peer review.

Conflicts of Interest: The authors have completed the ICMJE uniform disclosure form (available at https:// fomm.amegroups.com/article/view/10.21037/fomm-2044/coif). The series "Temporomandibular Joint Disorders Diagnosis and Management - What Does the Future Hold?" was commissioned by the editorial office without any funding or sponsorship. JP was a member of the Board of Directors of the Illinois Physical Therapy Foundation (unpaid), is a consultant for Myopain Seminars (paid), and receives conference and travel funding though Midwestern University. EK was a paid speaker at a conference, and receives conference and travel funding through the University of Minnesota. The authors have no other conflicts of interest to declare.

Ethical Statement: The authors are accountable for all aspects of the work in ensuring that questions related to the accuracy or integrity of any part of the work are appropriately investigated and resolved.

Open Access Statement: This is an Open Access article distributed in accordance with the Creative Commons Attribution-NonCommercial-NoDerivs 4.0 International License (CC BY-NC-ND 4.0), which permits the noncommercial replication and distribution of the article with the strict proviso that no changes or edits are made and the original work is properly cited (including links to both the formal publication through the relevant DOI and the license). See: https://creativecommons.org/licenses/by-nc-nd/4.0/.

\section{References}

1. Sturdivant J, Fricton JR. Physical therapy for temporomandibular disorders and orofacial pain. Curr Opin Dent 1991;1:485-96.

2. Kraus SL. Physical Therapy Management of Temporomandibular Disorders. In: Fonseca RJ, editor. Oral and Maxillofacial Surgery: Temporomandibular Disorders. Volume 4, 1st edition. WB Saunders Company; 2000:161-93.

3. McNeely ML, Armijo Olivo S, Magee DJ. A systematic review of the effectiveness of physical therapy interventions for temporomandibular disorders. Phys Ther 2006;86:710-25.

4. American Academy of Orofacial Pain, de Leeuw R, Klasser GD, editors. Orofacial Pain Guidelines for assessment, diagnosis, and management. 6th edition. Hanover Park, IL: Quintessence Publishing Co, Inc.; 2018.

5. Bond EC, Mackey S, English R, et al. The National Academies Collection: Reports funded by National Institutes of Health. Temporomandibular Disorders: Priorities for Research and Care. Washington (DC): National Academies Press (US); 2020:380.

6. Schiffman EL, Ahmad M, Hollender L, et al. Longitudinal Stability of Common TMJ Structural Disorders. J Dent Res 2017;96:270-6.

7. Tuijt M, Koolstra JH, Lobbezoo F, et al. Biomechanical modeling of open locks of the human temporomandibular joint. Clin Biomech (Bristol, Avon) 2012;27:749-53.

8. Tuijt M, Koolstra JH, Lobbezoo F, et al. How muscle relaxation and laterotrusion resolve open locks of the temporomandibular joint. Forward dynamic 3D-modeling of the human masticatory system. J Biomech 2016;49:276-83.

9. De Meurechy NKG, Loos PJ, Mommaerts MY. Postoperative Physiotherapy After Open Temporomandibular Joint Surgery: A 3-Step Program. J Oral Maxillofac Surg 2019;77:932-50.

10. Capan N, Esmaeilzadeh S, Karan A, et al. Effect of an early supervised rehabilitation programme compared with home-based exercise after temporomandibular joint condylar discopexy: a randomized controlled trial. Int J Oral Maxillofac Surg 2017;46:314-21.

11. Garrigós-Pedrón M, Elizagaray-García I, DomínguezGordillo AA, et al. Temporomandibular disorders: improving outcomes using a multidisciplinary approach. J Multidiscip Healthc 2019;12:733-47.

12. Gil-Martínez A, Paris-Alemany A, López-de-UraldeVillanueva I, et al. Management of pain in patients with temporomandibular disorder (TMD): challenges and solutions. J Pain Res 2018;11:571-87.

13. Dworkin SF, LeResche L. Research diagnostic criteria for temporomandibular disorders: review, criteria, examinations and specifications, critique. J Craniomandib Disord 1992;6:301-55.

14. Schiffman E, Ohrbach R, Truelove E, et al. Diagnostic Criteria for Temporomandibular Disorders (DC/TMD) for Clinical and Research Applications: recommendations of the International RDC/TMD Consortium Network and Orofacial Pain Special Interest Group. J Oral Facial 
Pain Headache 2014;28:6-27.

15. Harrison AL, Thorp JN, Ritzline PD. A proposed diagnostic classification of patients with temporomandibular disorders: implications for physical therapists. J Orthop Sports Phys Ther 2014;44:182-97.

16. Kraus S, Prodoehl J. Disc displacement without reduction with limited opening: A clinical diagnostic accuracy study. Physiother Theory Pract 2017;33:238-44.

17. Kraus SL. Characteristics of 511 patients with temporomandibular disorders referred for physical therapy. Oral Surg Oral Med Oral Pathol Oral Radiol 2014;118:432-9.

18. Teachey WS. Otolaryngic myofascial pain syndromes. Curr Pain Headache Rep 2004;8:457-62.

19. Gadotti IC, Hulse C, Vlassov J, et al. Dentists' Awareness of Physical Therapy in the Treatment of Temporomandibular Disorders: A Preliminary Study. Pain Res Manag 2018;2018:1563716.

20. Dalanon J, Ugalde RB, Catibod LD, et al. Comparative analysis of education, awareness, and knowledge of dentists and physical therapists in the treatment of temporomandibular disorders. Cranio 2020. [Epub ahead of print]. doi:10.1080/08869634.2020.1786332.

21. Klasser GD, Greene CS. Predoctoral teaching of temporomandibular disorders: a survey of U.S. and Canadian dental schools. J Am Dent Assoc 2007;138:231-7.

22. Prodoehl J, Kraus S, Klasser GD, et al.

Temporomandibular disorder content in the curricula of physical therapist professional programs in the United States. Cranio 2020;38:376-88.

23. Gadotti IC PT, PhD, Lakow A DPT, et al. Physical therapists' self-perceived adequacy of entry-level education and their current confidence levels with respect to temporomandibular disorders: A pilot study. Cranio 2020;38:312-9.

24. American Physical Therapy Association. Direct Access Utilization Survey Report. Alexandria, VA: American Physical Therapy Association; 2017.

25. Grondin F, Hall T, Laurentjoye M, et al. Upper cervical range of motion is impaired in patients with temporomandibular disorders. Cranio 2015;33:91-9.

26. De Leeuw R, Bertoli E, Schmidt JE, et al. Prevalence of post-traumatic stress disorder symptoms in orofacial pain patients. Oral Surg Oral Med Oral Pathol Oral Radiol Endod 2005;99:558-68.

27. Barnsteiner JH, Disch JM, Hall L, et al. Promoting interprofessional education. Nurs Outlook 2007;55:144-50.

28. Friction JR, Hathaway KM, Bromaghim C.
Interdisciplinary management of patients with TMJ and craniofacial pain: characteristics and outcome. J Craniomandib Disord 1987;1:115-22.

29. Silveira A, Gadotti IC, Armijo-Olivo S, et al. Jaw dysfunction is associated with neck disability and muscle tenderness in subjects with and without chronic temporomandibular disorders. Biomed Res Int 2015;2015:512792.

30. De Laat A, Meuleman H, Stevens A, et al. Correlation between cervical spine and temporomandibular disorders. Clin Oral Investig 1998;2:54-7.

31. Wänman A. The relationship between muscle tenderness and craniomandibular disorders: a study of 35-year-olds from the general population. J Orofac Pain 1995;9:235-43.

32. Inoue $\mathrm{E}$, Maekawa $\mathrm{K}$, Minakuchi $\mathrm{H}$, et al. The relationship between temporomandibular joint pathosis and muscle tenderness in the orofacial and neck/shoulder region. Oral Surg Oral Med Oral Pathol Oral Radiol Endod 2010;109:86-90.

33. von Piekartz H, Schwiddessen J, Reineke L, et al. International consensus on the most useful assessments used by physical therapists to evaluate patients with temporomandibular disorders: A Delphi study. J Oral Rehabil 2020;47:685-702.

34. Calixtre LB, Oliveira AB, de Sena Rosa LR, et al. Effectiveness of mobilisation of the upper cervical region and craniocervical flexor training on orofacial pain, mandibular function and headache in women with TMD. A randomised, controlled trial. J Oral Rehabil 2019;46:109-19.

35. Haiter-Neto F, Hollender L, Barclay P, et al. Disk position and the bilaminar zone of the temporomandibular joint in asymptomatic young individuals by magnetic resonance imaging. Oral Surg Oral Med Oral Pathol Oral Radiol Endod 2002;94:372-8.

36. Katzberg RW, Westesson PL, Tallents RH, et al. Anatomic disorders of the temporomandibular joint disc in asymptomatic subjects. J Oral Maxillofac Surg 1996;54:147-53; discussion 153-5.

37. Tasaki MM, Westesson PL, Isberg AM, et al. Classification and prevalence of temporomandibular joint disk displacement in patients and symptom-free volunteers. Am J Orthod Dentofacial Orthop 1996;109:249-62 .

38. Whittaker JL, Teyhen DS, Elliott JM, et al. Rehabilitative ultrasound imaging: understanding the technology and its applications. J Orthop Sports Phys Ther 2007;37:434-49.

39. Rathi S, Taylor NF, Gee J, et al. Measurement of glenohumeral joint translation using real-time ultrasound 
imaging: A physiotherapist and sonographer intra-rater and inter-rater reliability study. Man Ther 2016;26:110-6.

40. Ho KY, Laskowski B, Garcia D, et al. Measurement of anterior translation of the mandibular condyle using sonography. J Phys Ther Sci 2019;31:116-21.

41. Molinari F, Caresio C, Acharya UR, et al. Advances in quantitative muscle ultrasonography using texture analysis of ultrasound images. Ultrasound Med Biol 2015;41:2520-32.

42. Gijsbertse K, Goselink R, Lassche S, et al. Ultrasound Imaging of Muscle Contraction of the Tibialis Anterior in Patients with Facioscapulohumeral Dystrophy. Ultrasound Med Biol 2017;43:2537-45.

43. Baumer TG, Davis L, Dischler J, et al. Shear wave elastography of the supraspinatus muscle and tendon: Repeatability and preliminary findings. J Biomech 2017;53:201-4.

44. Turo D, Otto P, Shah JP, et al. Ultrasonic characterization of the upper trapezius muscle in patients with chronic neck pain. Ultrason Imaging 2013;35:173-87.

45. Dieterich AV, Andrade RJ, Le Sant G, et al. Shear wave elastography reveals different degrees of passive and active stiffness of the neck extensor muscles. Eur J Appl Physiol 2017;117:171-8.

46. Dieterich AV, Yavuz U, Petzke F, et al. Neck Muscle Stiffness Measured With Shear Wave Elastography in Women With Chronic Nonspecific Neck Pain. J Orthop Sports Phys Ther 2020;50:179-88.

47. Do TP, Heldarskard GF, Kolding LT, et al. Myofascial trigger points in migraine and tension-type headache. J Headache Pain 2018;19:84.

48. Blemker SS, Asakawa DS, Gold GE, et al. Image based musculoskeletal modeling: Applications, advances, and future opportunities. Journal of Magnetic Resonance Imaging 2007;25:441-51.

49. Dixit NN, McFarland DC, Fisher MB, et al. Integrated iterative musculoskeletal modeling predicts bone morphology following brachial plexus birth injury (BPBI). J Biomech 2020;103:109658.

50. Lawrence RL, Braman JP, Ludewig PM. The Impact of Decreased Scapulothoracic Upward Rotation on Subacromial Proximities. J Orthop Sports Phys Ther 2019;49:180-91.

51. Lawrence RL, Sessions WC, Jensen MC, et al. The effect of glenohumeral plane of elevation on supraspinatus subacromial proximity. J Biomech 2018;79:147-54.

52. Marra MA, Vanheule V, Fluit R, et al. A subject-specific musculoskeletal modeling framework to predict in vivo mechanics of total knee arthroplasty. J Biomech Eng 2015;137:020904.

53. Schiffman EL, Velly AM, Look JO, et al. Effects of four treatment strategies for temporomandibular joint closed lock. Int J Oral Maxillofac Surg 2014;43:217-26.

54. American Association of Oral and Maxillofacial Surgeons. Temporomandibualr Joint Surgery. In: Parameters of Care: AAOMS Clinical Practice Guidelines for Oral and Maxillofacial Surgery (AAOMS ParCare 2017). 6th edition. American Association of Oral and Maxillofacial Surgeons; 2017.

55. Dietrich EM, Griessinger N, Neukam FW, et al. Consultation with a specialized pain clinic reduces pain after oral and maxillofacial surgery. J Craniomaxillofac Surg 2017;45:281-9.

56. Calatayud J, Casaña J, Ezzatvar Y, et al. High-intensity preoperative training improves physical and functional recovery in the early post-operative periods after total knee arthroplasty: a randomized controlled trial. Knee Surg Sports Traumatol Arthrosc 2017;25:2864-72.

57. Sawaguchi A, Momosaki R, Hasebe K, et al. Effectiveness of preoperative physical therapy for older patients with hip fracture. Geriatr Gerontol Int 2018;18:1003-8.

58. Wang L, Lee M, Zhang Z, et al. Does preoperative rehabilitation for patients planning to undergo joint replacement surgery improve outcomes? A systematic review and meta-analysis of randomised controlled trials. BMJ Open 2016;6:e009857.

59. International Association for the Study of Pain. IASP Interprofessional Pain Curriculum Outline 2020. Available online: https://www.iasp-pain.org/Education/ CurriculumDetail.aspx? ItemNumber $=2057$

60. Morton DL, Sandhu JS, Jones AK. Brain imaging of pain: state of the art. J Pain Res 2016;9:613-24.

61. Rammelsberg P, LeResche L, Dworkin S, et al. Longitudinal outcome of temporomandibular disorders: a 5-year epidemiologic study of muscle disorders defined by research diagnostic criteria for temporomandibular disorders. J Orofac Pain 2003;17:9-20.

62. La Touche R, Paris-Alemany A, Hidalgo-Perez A, et al. Evidence for Central Sensitization in Patients with Temporomandibular Disorders: A Systematic Review and Meta-analysis of Observational Studies. Pain Pract 2018;18:388-409.

63. Finnerup NB, Haroutounian S, Kamerman P, et al. Neuropathic pain: an updated grading system for research and clinical practice. Pain 2016;157:1599-606.

64. Latremoliere A, Woolf CJ. Central sensitization: a 
generator of pain hypersensitivity by central neural plasticity. J Pain 2009;10:895-926.

65. Lira MR, Lemes da Silva RR, Bataglion C, et al. Multiple diagnoses, increased kinesiophobia? - Patients with high kinesiophobia levels showed a greater number of temporomandibular disorder diagnoses. Musculoskelet Sci Pract 2019;44:102054.

66. Visscher CM, Ohrbach R, van Wijk AJ, et al. The Tampa Scale for Kinesiophobia for Temporomandibular Disorders (TSK-TMD). Pain 2010;150:492-500.

67. Gil-Martínez A, Grande-Alonso M, López-de-UraldeVillanueva I, et al. Chronic Temporomandibular Disorders: disability, pain intensity and fear of movement. J Headache Pain 2016;17:103.

68. Gil-Martínez A, Grande-Alonso M, La Touche R, et al. Psychosocial and Somatosensory Factors in Women with Chronic Migraine and Painful Temporomandibular Disorders. Pain Res Manag 2016;2016:3945673.

69. La Touche R, Paris-Alemany A, Gil-Martínez A, et al. Masticatory sensory-motor changes after an experimental chewing test influenced by pain catastrophizing and neckpain-related disability in patients with headache attributed to temporomandibular disorders. J Headache Pain 2015;16:20.

70. Vlaeyen JW, Linton SJ. Fear-avoidance and its consequences in chronic musculoskeletal pain: a state of the art. Pain 2000;85:317-32.

71. Crombez G, Eccleston C, Baeyens F, et al. Attention to chronic pain is dependent upon pain-related fear. J Psychosom Res 1999;47:403-10.

72. Leeuw M, Goossens ME, Linton SJ, et al. The fearavoidance model of musculoskeletal pain: current state of scientific evidence. J Behav Med 2007;30:77-94.

73. Swinkels-Meewisse IE, Roelofs J, Schouten EG, et al. Fear of movement/(re)injury predicting chronic disabling low back pain: a prospective inception cohort study. Spine (Phila Pa 1976) 2006;31:658-64.

74. Cresswell C, Galantino ML, Myezwa H. The prevalence of fear avoidance and pain catastrophising amongst patients with chronic neck pain. S Afr J Physiother 2020;76:1326.

75. Andersen TE, Karstoft KI, Brink O, et al. Paincatastrophizing and fear-avoidance beliefs as mediators between post-traumatic stress symptoms and pain following whiplash injury - A prospective cohort study. Eur J Pain 2016;20:1241-52.

76. France CR, Thomas JS. Virtual immersive gaming to optimize recovery (VIGOR) in low back pain: A phase II randomized controlled trial. Contemp Clin Trials
2018;69:83-91.

77. Thomas JS, France CR, Applegate ME, et al. Feasibility and Safety of a Virtual Reality Dodgeball Intervention for Chronic Low Back Pain: A Randomized Clinical Trial. J Pain 2016;17:1302-17.

78. Matheve T, Bogaerts K, Timmermans A. Virtual reality distraction induces hypoalgesia in patients with chronic low back pain: a randomized controlled trial. J Neuroeng Rehabil 2020;17:55.

79. Lohse KR, Sherwood DE, Healy AF. On the advantage of an external focus of attention: a benefit to learning or performance? Hum Mov Sci 2014;33:120-34.

80. Lohse KR. The influence of attention on learning and performance: pre-movement time and accuracy in an isometric force production task. Hum Mov Sci 2012;31:12-25.

81. Yilmaz Yelvar GD, Çırak Y, Dalkılınç M, et al. Is physiotherapy integrated virtual walking effective on pain, function, and kinesiophobia in patients with non-specific low-back pain? Randomised controlled trial. Eur Spine J 2017;26:538-45.

82. Moseley GL. A pain neuromatrix approach to patients with chronic pain. Man Ther 2003;8:130-40.

83. Butler D, Moseley GL. Explain Pain. 2nd edition. NOI Group; 2013.

84. Louw A, Zimney K, Puentedura EJ, et al. The efficacy of pain neuroscience education on musculoskeletal pain: A systematic review of the literature. Physiother Theory Pract 2016;32:332-55.

85. Fernandez de las Penas C, Cleland J, Dommerhalt J. Manual Therapy for Musculoskeletal Pain Syndromes: an evidence- and clinical-informed approach. 1st edition. Churchill Livingstone; 2015.

86. Louw A, Diener I, Landers MR, et al. Preoperative pain neuroscience education for lumbar radiculopathy: a multicenter randomized controlled trial with 1-year follow-up. Spine (Phila Pa 1976) 2014;39:1449-57.

87. Louw A, Puentedura EJ, Diener I, et al. Pain neuroscience education: Which pain neuroscience education metaphor worked best? S Afr J Physiother 2019;75:1329.

88. Wood L, Hendrick PA. A systematic review and metaanalysis of pain neuroscience education for chronic low back pain: Short-and long-term outcomes of pain and disability. Eur J Pain 2019;23:234-49.

89. Louw A, Diener I, Landers MR, et al. Three-year followup of a randomized controlled trial comparing preoperative neuroscience education for patients undergoing surgery for lumbar radiculopathy. J Spine Surg 2016;2:289-98. 
90. Louw A, Butler DS, Diener I, et al. Development of a preoperative neuroscience educational program for patients with lumbar radiculopathy. Am J Phys Med Rehabil 2013;92:446-52.

91. Louw A, Diener I, Butler DS, et al. Preoperative education addressing postoperative pain in total joint arthroplasty: review of content and educational delivery methods. Physiother Theory Pract 2013;29:175-94.

92. Louw A, Zimney K, Reed J, et al. Immediate preoperative outcomes of pain neuroscience education for patients undergoing total knee arthroplasty: A case series. Physiother Theory Pract 2019;35:543-53.

93. Gallagher L, McAuley J, Moseley GL. A randomizedcontrolled trial of using a book of metaphors to reconceptualize pain and decrease catastrophizing in people with chronic pain. Clin J Pain 2013;29:20-5.

doi: $10.21037 /$ fomm-20-44

Cite this article as: Prodoehl J, Kahnert E. Physical therapy for temporomandibular disorders: evidence-based improvements and enhancements for diagnosis and management. Front Oral Maxillofac Med 2022;4:16.
94. Blanchard L, Goostree S, Duncombe A. Graded exposure and orthopedic manual physical therapy for kinesiophobia and function in chronic temporomandibular dysfunction: A case report. Cranio 2020. [Epub ahead of print]. doi:10. 1080/08869634.2020.1779483.

95. Louw A, Puentedura EJ, Reed J, et al. A controlled clinical trial of preoperative pain neuroscience education for patients about to undergo total knee arthroplasty. Clin Rehabil 2019;33:1722-31.

96. Polli A, Ickmans K, Godderis L, et al. When Environment Meets Genetics: A Clinical Review of the Epigenetics of Pain, Psychological Factors, and Physical Activity. Arch Phys Med Rehabil 2019;100:1153-61.

97. Jaenisch R, Bird A. Epigenetic regulation of gene expression: how the genome integrates intrinsic and environmental signals. Nat Genet 2003;33 Suppl:245-54. 